\title{
A cricket-song simulator using the 68705 single-chip microcomputer
}

\author{
D. JAMES CAMPBELL and JOHN FOREST \\ The University of Wollongong, Wollongong, New South Wales, Australia
}

\begin{abstract}
A cricket-song simulator is described. The device uses a waveform generator to produce a song carrier frequency that is modulated by the 68705 microcomputer. Sixteen different songs can be stored in memory.
\end{abstract}

The cricket song simulator described here was developed from a 6502 -based system previously described by Campbell and Loher (1983). It was designed to generate simulated cricket songs, with specific parameters required for a series of field and laboratory experiments, but it can be used for a variety of purposes for which a particular pulsed output pattern is required. Walker (1982) has used a similar device in conjunction with sound traps for monitoring migrations of crickets. In the system described here, an 8038 waveform generator produces the song carrier frequency that is modulated by the 68705 microcomputer. The output is amplified with a 386 audio amplifier, and the simulated song is transmitted from a piezoelectric tweeter. Up to 16 different songs (0-15) can be stored in computer memory as song tables, and each song can be selected by means of four toggle switches that specify the song number in binary.

Use of the 68705 has a number of advantages. It has the essentials of a complete microcomputer, such as input/output ports, a CPU, and RAM. In addition, there is user-programmable EPROM and a bootstrap program in ROM that is used for easy EPROM programming. All of these features are on one chip, so there are obvious benefits if compactness and low costs in a dedicated system are considerations. Another advantage of the 68705 is that the EPROM is nonvolatile and the device functions immediately on power-up without the need for loading a program. Also, the program is not lost in the event of a power failure.

The 68705 is available in both HMOS (MC68705P3), which requires a relatively high operating current but is well suited to operation using a power supply, and in CMOS (MC1468705F2), which is suitable for battery operation. The instruction set is the same for both, but unfortunately the pin configurations and the bootstrap routines are not. The HMOS 68705 is used in the unit described here; however, it should be relatively simple to make the necessary modifications if a CMOS batteryoperated system is required. Full technical data for both chips are available from Motorola distributors.

We thank John Murray for the diagram work. The authors' mailing address is: Department of Biology, The University of Wollongong, P.O. Box 1144, Wollongong, New South Wales 2500, Australia.

\section{SONG TABLES}

The song tables are the means by which the user specifies the parameters of the simulated songs required. The tables are in hexadecimal notation and control the durations of consecutive output levels of the song modulator. The initial level is set LO, the second level HI, the third, LO, and so forth. Although the duration of each level is specified by the table elements, as in the earlier version (Campbell \& Loher, 1983), the level (HI or LO) is not. This makes the song tables shorter and simpler to write. For example, initial elements 0606 in a table would produce a pulse of duration 6 unit intervals $L O$ and 6 unit intervals HI. The unit interval is the same for both versions $(1,160 \mu \mathrm{sec})$.

A segment of the table consists of one or more elements demarked by an FE flag and followed by the number of repeats of the segment required by the user. Thus 0606 FE $0 \mathrm{C}$ would produce 12 pulses with a periodicity of $(6 \times 1,160) \times 2 \mu \mathrm{sec}$. The first segment can be followed by a second, a third, and so forth, each demarked by an FE flag and the number of repeats required.

All the segments of a table constitute the phrase, the end of which is denoted by an FF flag. The phrase is repeated indefinitely: for example, $2020 \mathrm{FE} 061010$ FE 1E A0 FE $01 \mathrm{FF}$. This represents a complete song
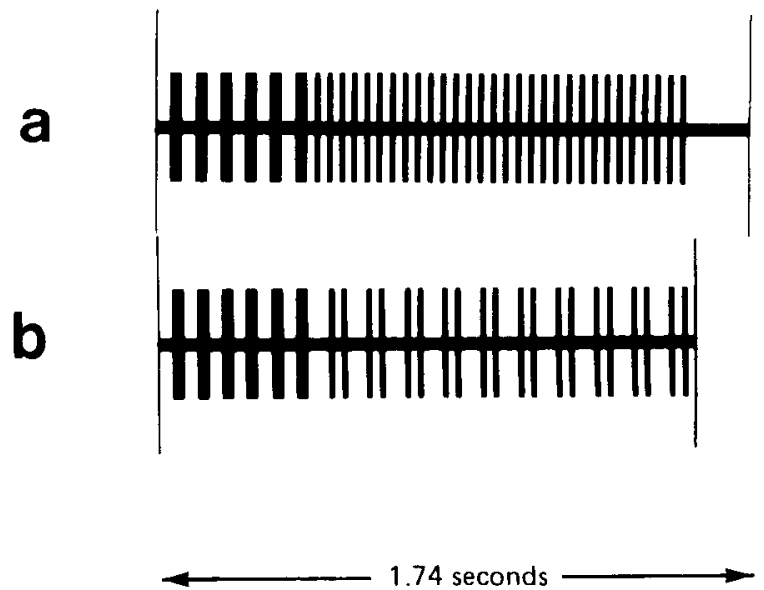

Figure 1. Simulations of the calling songs of two field crickets: (a) Teleogryllus commodus and (b) Teleogryllus oceanicus. 
table for the calling song of the cricket Teleogryllus commodus (see Figure la). It has the same parameters as those previously described (Campbell \& Loher, 1983).

An extended pause can be set in the song table as a single (LO) element in a segment, as was done in the last (third) segment of the preceding song table. In this case, the level is not toggled. For example, 01 FE 01 FF results in a continuous $\mathbf{L O}$.

A further advantage of this version of the song modulator is that complex songs are readily specified by having the possibility of more than two elements in a segment (the limit in the earlier version): for example, 20 . $20 \mathrm{FE} 0630101010 \mathrm{FE} 0 \mathrm{~A} 10 \mathrm{FE} 01 \mathrm{FF}$. In this case, the second segment has four elements (30 101010$)$. This . is a table for the calling song of Teleogryllus oceanicus . : (see Figure 1b).

\section{THE PROGRAM}

\section{Development}

It is not possible to experiment with the 68705 or any EPROM device without peripherals. Therefore, program development and evaluation need to be done on a unit with programmable RAM, a keyboard, and a display unit. In this case, we utilized the KIM-1 for program development using an instruction subset common to both the 6502 and the 68705. Timing loops were adjusted to compensate for the differences in the cycle times of the two systems. The 68705 has no indirect indexed addressing, and, therefore, the indexed indirect mode was used throughout where indirect addressing was required $(6502$ terminology). This has limited the memory available for song tables to one page (0300-03FF), but the tables for most song simulations require less than 16 entries each, so this limitation is not a problem.

Programs are in assembly language and all opcodes and addresses are, therefore, in hexadecimal notation. (A full listing with comments of the program for the KIM-1 is available on request.) The hexadecimal listing for the equivalent 68705 program is given in Table 1 . This represents a one-to-one translation of the KIM-1 program. The opcodes for the 68705 instruction set are given in the Motorola data sheets. The main difference in the two systems (requiring care in the translation) is that the operand addresses are specified high order first for the 68705 and low order first in the 6502 system.

\section{Ports}

In this new program, ports $B$ and $C$ of the 68705 correspond functionally to ports $\mathrm{B}$ and $\mathrm{A}$, respectively, of the KIM-1. The output is from PBO. Levels (HI or LO) of the song elements are determined by the initial condition of PB1 (pulled LO in our circuit). Output is terminated if either PB2 or PB3 is pulled LO. Choice of song number is by means of four toggle switches wired from $\mathrm{PC} 0, \mathrm{PC} 1, \mathrm{PC} 2, \mathrm{PC} 3$ to ground (PA0 to PA3 in the KIM1). These specify $0,0,0,0$ when closed and 1, 2, 4, 8 when open. Thus, any song number between 0 and 15 can be selected by the appropriate arrangement of these four switches. The song number will not change for new switch positions until PB2 or PB3 is toggled LO and then $\mathrm{HI}$ again or until the 68705 is reset (by toggling pin 28 $\mathrm{LO}$ and then $\mathrm{HI}$ again, or by powering up). The unmodulated carrier frequency is produced while the reset pin 28 is pulled LO.

Table 1

Listing of the 68705 Song Simulator Program and Ancillary Data

91906 B⿺

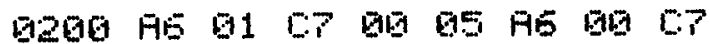

$\begin{array}{lllllllll}0209 & 06 & 05 & 04 & 01 & \text { A4 } & 02 & 46\end{array}$

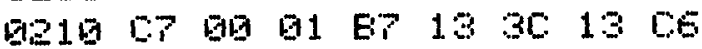

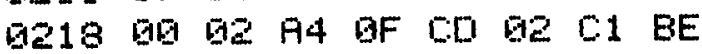

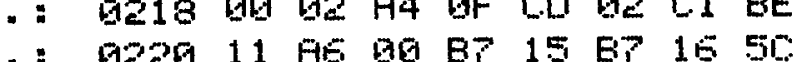

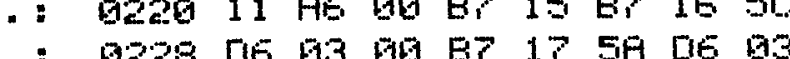

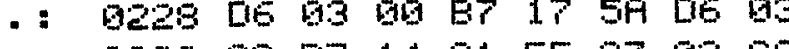

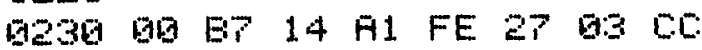

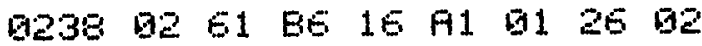

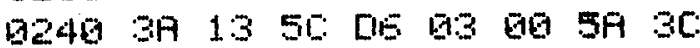

024815 E1 1527 GS $5 \mathrm{~A}$ 3A 16

0250 26 FB dC 92 27 50 50 as

G250 03 GQ H1 FF 27 D. CO 02

926021 3E 15 35 13 BE 13 CP

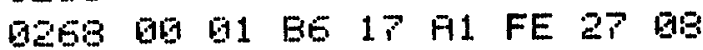

Q270 AE 99 BP 19 3F 19 28 FC

G273 AS $5 E$ BP 18 SH 13 26 FL

9230 उA 14 27 BE $A 5$ 13 ET 18

Q2BB $3 A$ 18 26 FE EL BC 7350

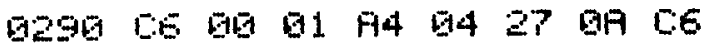

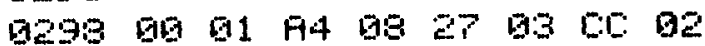

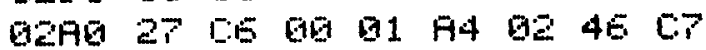

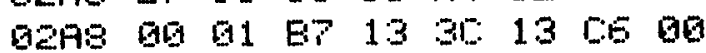

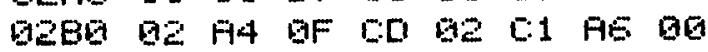

Q2EB ET $15 \mathrm{BP}$ 1E EE 11 CO 02

ㅁ200 9098 BP 19 BS 1997 [16

Q203 Q2 EG ET 11 पIE G2 E1 ET

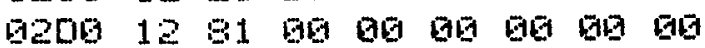

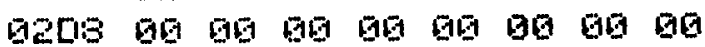

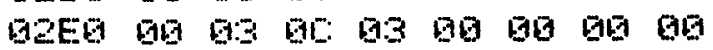

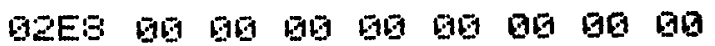

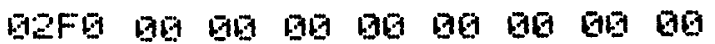

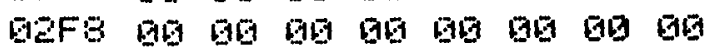

EOBg 20 20 FE DE 10 19 FE IE

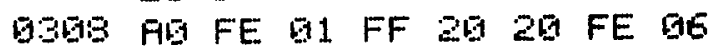

D310 30 10 19 10 FE BA 10 FE

QI18 81 FF

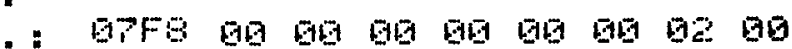




\section{Examples}

Table 1 includes the program (0200-02D1) and the pointers for the starting addresses for the song tables of Song 0 ( $T$. commodus) and Song 1 ( $T$. oceanicus) at 02E0,02E1 and 02E2,02E3 (low order first). The song tables for these two songs are at $0300-030 \mathrm{~B}$ and $030 \mathrm{C}$ 0319. The last two locations, 07FE and 07FF (in the last line of Table 1), contain the reset vectors that point to the starting address of the program. The starting address pointers for all 16 song tables, if all are used, are specified by the user between 02E0 and 02FF, and data for the song tables are entered between 0300 and 03FF.

\section{EPROM PROGRAMMING THE 68705}

The song simulator program (Table 1), the song tables and addresses, and the reset vectors are transferred from an external 2716 EPROM to the 68705 EPROM using the bootstrap ROM of the 68705 . The circuit diagram for the hardware required for the transfer and an explanation of its operation are provided in the Motorola data sheets; therefore, details are not included here.

Programming the external 2716 EPROM was achieved using a Jason-Ranheim 'Promenade" programmer. 'This fits into the user port of the Commodore-64 computer and is controlled by software that comes with the device. The procedure is to transfer the song simulator program plus ancillary data as a block stored anywhere convenient in the C-64 user RAM (e.g., 2000-27FF) to the 2716 EPROM. The "Promenade" manual describes various ways of doing this. Transferring the program and data into the C-64 user RAM is probably most easily done by using a monitor.

\section{HARDWARE}

The hardware schematic is given in Figure 2. We used a Kambrook programmable timer to switch the song simulator on and off at specified times. It is available at many retail outlets as a digital time switch for domestic appliances. It was chosen because it has a $12-\mathrm{V}$ dc supply, an LCD clock with battery backup, up to eight time settings, and, most importantly, a TTL output from the control chip. The song simulator hardware is housed in a small jiffy box glued over the power-out socket (not required) of the Kambrook timer to provide a very compact unit measuring about $8 \times 6 \times 15 \mathrm{~cm}$. The TTL line from the timer is wired to PB3 of the 68705 . The pull-up on this line, shown in Figure 2, might not be necessary, depending on the characteristics of the timer. The 12-V supply line is used to run the 8038 waveform generator and the 386 audio amplifier. This is sufficient to give a modu-

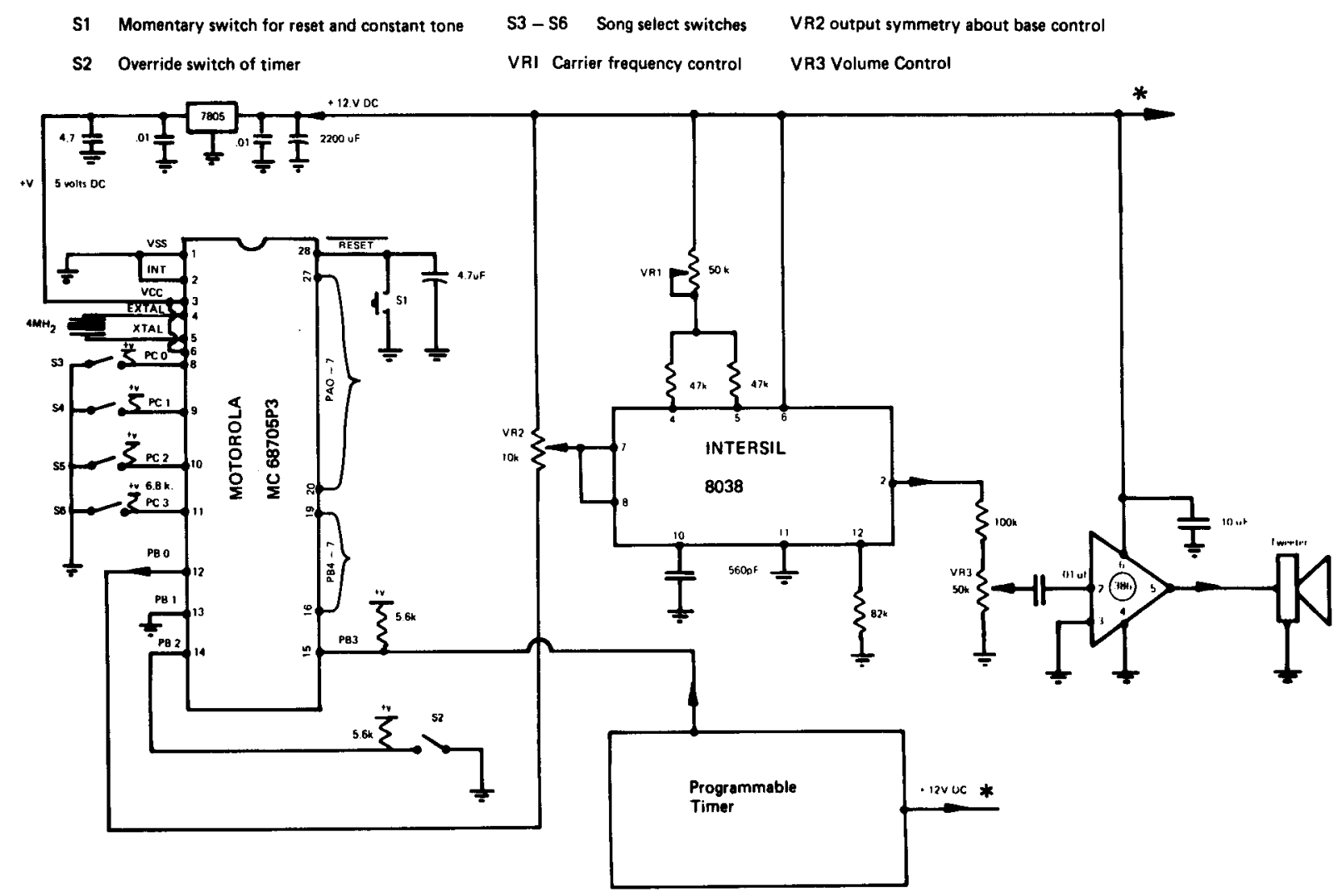

Figure 2. Schematic for the cricket-song simulator. 
lated sine wave output of about $9 \mathrm{~V}$ peak to peak without clipping. VCC of the 68705 requires a 5-V supply, which is obtained via a $5-\mathrm{V}$ regulator from the $12-\mathrm{V}$ supply. The 8038 has been wired to provide a frequency range of approximately $2-6 \mathrm{kHz}$ using VRI.

\section{REFERENCES}

CAMPBell, D. J., \& LOHER, W. (1983). A microcomputer-based modulator for simulating insect songs and the response of crickets to an artificial calling song. Behavior Research Methods \& Instrumentation, 15, 538-541.
WALKER, T. J. (1982). Sound traps for sampling mole cricket flights (Orthoptera: Gryllotalpidae: Scapteriscus). The Florida Entomologist, 65, 105-110.

\section{NOTE}

1. The "Promenade" EPROM programmer is currently available from Jason-Ranheim, 580 Parrott Street, San Jose, CA 95112.

(Manuscript received March 21, 1986; revision accepted for publication June 29,1986 .) 